\title{
Regeneração de Protium pallidum Cuatrec. em clareiras originadas da exploração madeireira seletiva na Amazônia Oriental Brasileira
}

\author{
Regeneration of Protium pallidum Cuatrec. in clearings originated from selective logging in the \\ Eastern Brazilian Amazon
}

Regeneración de Protium pallidum Cuatrec. en desmontes originados por la tala selectiva en el este de la Amazonía brasileña

\author{
Stone César Cavalcante da Costa \\ ORCID: https://orcid.org/0000-0003-1894-8118 \\ Secretaria de Estado de Meio Ambiente e Sustentabilidade, Brasil \\ E-mail: stonecosta@yahoo.com.br \\ Fernando Cristóvam da Silva Jardim \\ ORCID: https://orcid.org/0000-0002-9855-759X \\ Universidade Federal Rural da Amazônia, Brasil \\ E-mail: fernando.jardim@ufra.edu.br \\ Francimary da Silva Carneiro \\ ORCID: https://orcid.org/0000-0002-1693-8779 \\ Secretaria de Estado de Meio Ambiente e Sustentabilidade, Brasil \\ E-mail: francimarycarneiro@gmail.com \\ Marcio Braga Amorim \\ ORCID: https://orcid.org/0000-0001-7508-2766 \\ Secretaria de Estado de Meio Ambiente e Sustentabilidade, Brasil \\ E-mail: marciobamorim@gmail.com \\ Klewton Adriano Oliveira Pinheiro \\ ORCID: https://orcid.org/0000-0003-2696-4249 \\ Instituto Federal de Educação, Ciências e Tecnologia do Pará, Brasil \\ E-mail: klewton.pinheiro@gmail.com
}

\begin{abstract}
Resumo
O objetivo desta pesquisa foi avaliar a dinâmica de regenerantes de Protium pallidum Cuatrec., em clareiras resultantes da exploração madeireira seletiva, foram utilizadas nove clareiras neste estudo, com tamanho variando entre $231 \mathrm{~m} 2$ e $748 \mathrm{~m} 2$. Cada clareira teve seu centro determinado e a partir da borda, foram marcadas quatro faixas de 10m x 50m nas direções Norte, Sul, Leste e Oeste. Para a avaliação da regeneração natural, foram implantadas três parcelas de $2 \mathrm{~m} \times 2 \mathrm{~m}$ em cada faixa, nas distâncias de 0,20 e $40 \mathrm{~m}$ respectivamente. utilizou-se os modelos matemáticos denominados Taxa de Regeneração Natural (TR\%), Ingresso (I\%) e Mortalidade (M\%). Foi feita a análise de variância de três fatores. Não houve diferença significativa dos valores de TR\% tanto em função das distâncias, quanto das direções e também em função dos anos, porém essa TR\% teve os maiores valores registrados para a direção Sul e na parcela a 40m de distância no interior do sub-bosque.
\end{abstract}

Palavras-chave: Dinâmica florestal; Ingresso e recrutamento; Sobrevivência de mudas; Incremento florestal.

\begin{abstract}
The objective of this research was to evaluate the dynamics of Protium pallidum Cuatrec. Regenerants, in clearings resulting from selective logging, nine clearings were used in this study, ranging in size from $231 \mathrm{~m} 2$ to $748 \mathrm{~m} 2$. Each clearing had its center determined and from the edge four strips of $10 \mathrm{~m}$ x $50 \mathrm{~m}$ were marked in the North, South, East and West directions. For the evaluation of natural regeneration, three plots of $2 \mathrm{~m} \times 2 \mathrm{~m}$ were implanted in each strip, at distances of 0,20 and 40m respectively. the mathematical models called Natural Regeneration Rate (TR\%), Ingresso (I\%) and Mortality (M\%) were used. Three-way analysis of variance was performed. There was no significant difference in the TR\% values as a function of distances, as well as directions and also as a function of years, however this TR\% had the highest values recorded for the South direction and in the plot 40m away in the interior of the subgrove.
\end{abstract}

Keywords: Forest dynamics; Entry and recruitment; Seedling survival; Forest increment.

\section{Resumen}

La dinámica y diversidad de los bosques tropicales están influenciadas por varios eventos naturales. Los estudios sobre estos eventos son esenciales para comprender las posibles direcciones de la sucesión forestal y pueden ser 
indicadores importantes de la sostenibilidad de los bosques tropicales y los sistemas de manejo. Este estudio tuvo como objetivo evaluar la dinámica de individuos de regeneración natural (DAP $<5 \mathrm{~cm}$ ) de Protium pallidum Cuatrec., Bajo la influencia de claros, mediante la determinación de las tasas de entrada, mortalidad y regeneración natural, en un tramo de bosque tropical primario. explorado en el Estado de Pará, Brasil. A partir de esta exploración se originaron varios claros, de los cuales nueve se utilizaron en este estudio, que varían en tamaño desde $231 \mathrm{~m} 2$ a 748m2. Cada claro tenía su centro determinado y desde el borde se marcaron cuatro franjas de $10 \mathrm{mx} 50 \mathrm{~m}$ en las direcciones norte, sur, este y oeste. Para la evaluación de la regeneración natural, se implantaron tres parcelas de $2 \mathrm{~m} \mathrm{x}$ $2 \mathrm{~m}$ en cada franja, a distancias de 0,20 y $40 \mathrm{~m}$ respectivamente. Posteriormente se utilizaron modelos matemáticos denominados Tasa de Regeneración Natural (TR\%), Ingresso (I\%) y Mortalidad (M\%). Los datos fueron analizados en el programa SYSTAT 10, mediante el análisis de varianza de tres factores (direcciones, distancias y el período de tres años). No hubo diferencia significativa en los valores de TR\% tanto en función de distancias, como de direcciones y también en función de años, sin embargo este TR\% tuvo los valores más altos registrados para la dirección Sur y en la parcela $40 \mathrm{~m}$ dentro de la sub-arboleda.

Palabras clave: Dinámica forestall; Claros; Regeneración natural.

\section{Introdução}

A dinâmica e a diversidade de florestas tropicais são influenciadas por eventos naturais que fazem com que a floresta se pareça com um mosaico composto de árvores de vários tamanhos e idades (Brokaw \& Scheiner, 1989; Brown, 1993; Denslow, 1980; Whitmore, 1991). Isso acontece porque as mudas de diferentes espécies de árvores podem alcançar ótimo crescimento sob diferentes regimes de luz em diferentes tamanhos de clareiras (Canham, 1989; Clark, 1990; Whitmore, 1991).

Além disso alterações causadas por cortes seletivos resultam em mudanças na penetração de luz solar no sub-bosque, geralmente até mesmo longe das clareiras, podendo afetar a distribuição e o crescimento das espécies, muitas das quais são de interesse comercial (Johns, 1997; Vieira, 1995).

Dessa maneira, estudos de dinâmica de clareiras e efeito de borda são fundamentais para entender as causas e possíveis direções da sucessão florestal e podem ser indicadores importantes da sustentabilidade das florestas tropicais e sistemas de manejo (Johns, 1997; Whitmore, 1991).

A avaliação da qualidade dos sítios de regeneração e a quantificação do estoque de indivíduos jovens são primordiais para a implementação dos níveis de intensidade de manejo, para manter o contingente populacional reprodutiva e ecologicamente viável (Vieira \& Hosokawa, 1989).

É crescente a demanda pelos recursos florestais, tornando o ato da exploração uma ameaça para a conservação desses bens naturais. O potencial madeireiro das florestas tropicais está sendo cada vez mais afetado pelo desordenado e acelerado consumo, sendo que as técnicas de manejo ainda são pouco utilizadas atualmente, não só pelo total descaso dos que fazem uso desse potencial, mas também pela falta de conhecimento acerca do comportamento das espécies que compõem a flora (Vasconcelos, 2004).

Este estudo teve como objetivo avaliar a dinâmica dos indivíduos da regeneração natural (DAP $<5 \mathrm{~cm}$ ) de Protium pallidum Cuatrec., sob a influência de clareiras, através da determinação de ingresso, mortalidade e taxa de regeneração natural (TR\%), em um trecho de floresta tropical primária explorada seletivamente no Município de Moju-PA.

Uma vez que a regeneração natural das espécies de florestas tropicais de terra firme depende, de uma forma geral, das dinâmicas das clareiras naturais e as subsequentes alterações sob os regimes de fatores ambientais (notadamente luz e umidade) (Johns, 1997; Whitmore, 1991), alterações causadas por cortes seletivos resultam em mudanças na penetração de luz solar no sub-bosque e efeitos indiretos sobre a umidade e outros fatores ambientais importantes para a regeneração. As clareiras formadas durante as intervenções afetam a distribuição e crescimento de muitas espécies, com possíveis implicações sobre padrões quali-quantitativos da distribuição das espécies arbóreas, resultando em alterações do valor da floresta residual nos futuros ciclos de corte (Johns, 1997; Vieira, 1995).

A dinâmica e diversidade de florestas tropicais são influenciadas por eventos naturais que fazem com que a floresta se pareça com um mosaico composto de árvores de vários tamanhos e idades (Brokaw \& Scheiner, 1989; Brown, 1993; Denslow, 
1980; Whitmore, 1991). Isso acontece porque as mudas de diferentes espécies de árvores podem alcançar ótimo crescimento sob diferentes regimes de luz em diferentes tamanhos de clareiras (Canham, 1989; Clark, 1990; Whitmore, 1991).

\section{Metodologia}

\section{Caracterização da área de estudo}

A área de estudo localiza-se no Campo Experimental da Embrapa Amazônia Oriental, situado no km 30, à margem esquerda da Rodovia PA 150, no município de Moju, na microrregião 18 do Estado do Pará, distando 34 km da sede do município de Moju e $115 \mathrm{~km}$ da cidade de Belém (Figura 1).

O Campo Experimental está situado entre as coordenadas geográficas de $2^{\circ} 7^{\prime} 57,03^{\prime \prime}$ e $2^{\circ} 18^{\prime} 28,57^{\prime \prime}$ de latitude Sul e $48^{\circ} 47^{\prime} 10,92^{\prime \prime}$ e $48^{\circ} 55^{\prime}$ 0,35" de longitude a Oeste do meridiano de Greenwich, possuindo uma área de 1.059 hectares, na qual foram selecionados 200 ha, nos quais foi realizada uma exploração madeireira (Carneiro et al, 1919).

Figura 1 - Localização da área de estudo, destacando o Estado do Pará (A), o Município de Moju (B) e a Estação Experimental da Embrapa (C). (Fonte: UAS, Museu Paraense Emílio Goeldi, janeiro 2006).
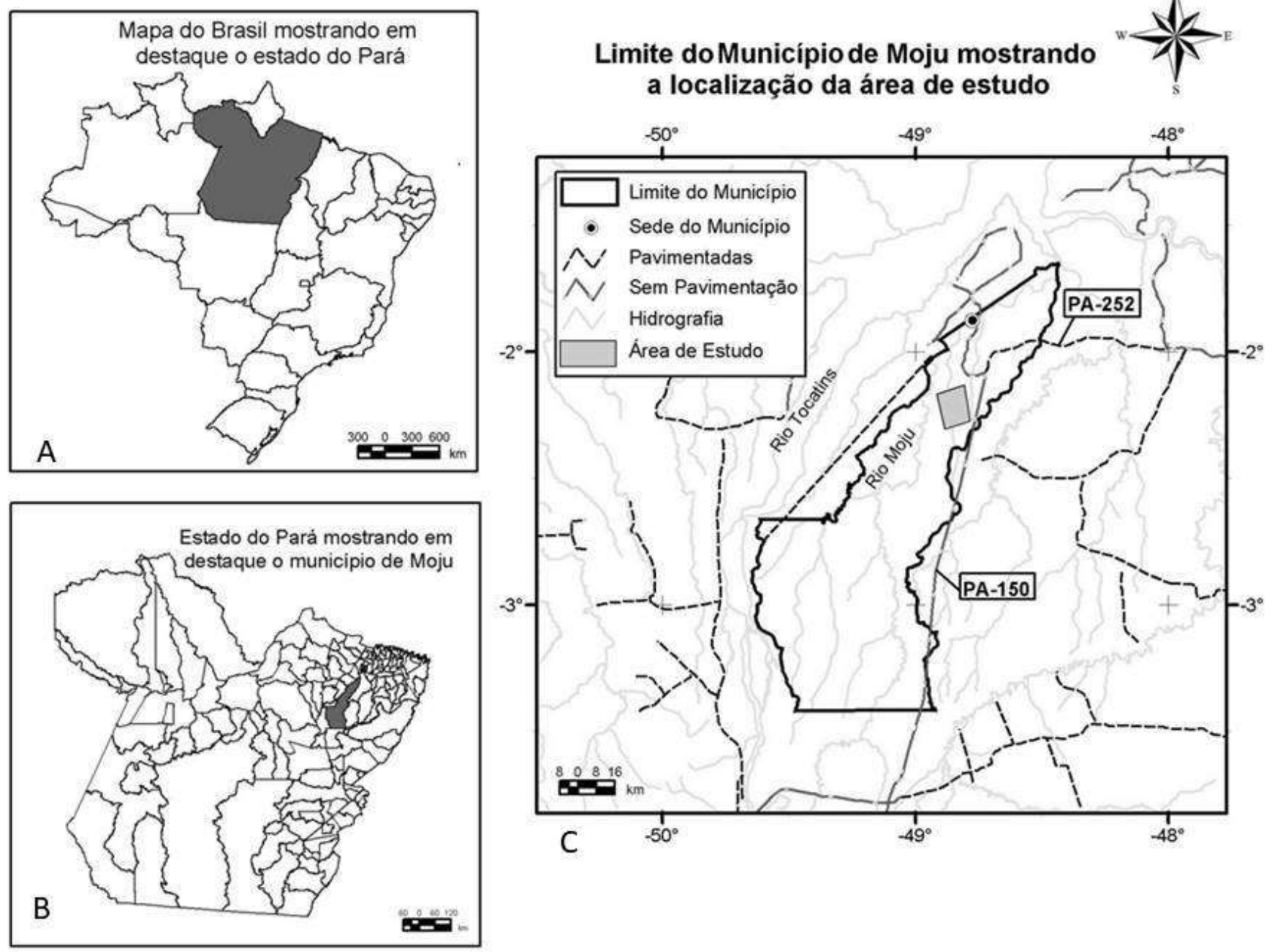

Fonte: Autores.

O clima da região é Ami, segundo a classificação de Köppen, com temperatura média anual oscilando entre 25o C e 27o C e precipitação anual entre 2000 mm e 3000 mm, com distribuição irregular e com ocorrência de dois períodos de chuva, 
o mais chuvoso abrangendo o período de janeiro a junho, concentrando cerca de $70 \%$ da precipitação anual. A insolação mensal varia entre $148,0 \mathrm{~h}$ e $275,8 \mathrm{~h}$ (Santos et al. 1985).

O relevo é plano a suavemente ondulado, com pequenos desnivelamentos que variam de $0 \%$ a $3 \%$, e de $3 \%$ a $8 \%$ de declive, respectivamente, onde predominam solos bem drenados, classificados como Latossolos Amarelos e Argissolos Amarelos típicos e com suas fases pedregosas.

A cobertura vegetal da região, segundo a classificação adotada pela Embrapa em 1979, está representada por duas formações florestais bem definidas, que são:

a) Floresta equatorial subperenifólia: tem como característica principal, ser formada em sua maioria por exemplares de grande porte, em torno de 25 a 35 metros de altura, com copas grandes e irregulares, formando muitas vezes uma cobertura contínua e apresentando um sub-bosque bem desenvolvido, com espécies características da formação, com presença de algumas palmáceas. As espécies de maior importância encontradas nessa formação foram: acapu - Vouacapoua americana Ducke, maçaranduba - Manilkara huberi Ducke, mata-matá - Eschweilera spp., quaruba - Vochysia guianensis Aubl., angelim - Dinizia excelsa Ducke, pau-d'arco - Tabebuia serratifolia (Vahl) Nichols, marupá - Simaruba amara Aubl., freijó - Cordia goeldiana Hub., bacuri - Platonia insignis Mart., cumaru - Dipteryx spp., jatobá - Hymenaea courbaril L., cupiúba - Goupia glabra Aubl (Santos et al., 2003).

b) Floresta equatorial higrófila de várzea: é uma formação cuja característica principal é encontrar-se sujeita a influência maior de flutuação dos níveis das águas nos períodos de cheias e vazantes. Sua ocorrência está estreitamente relacionada com o relevo condicionada à drenagem. É encontrada nas várzeas do Rio Ubá e em baixios alimentados através de igarapés no período das inundações. As espécies registradas são em maiores percentagens de madeiras moles, com baixo valor comercial, exceto a andiroba - Carapa guianensis Aublet, açacu - Hura crepitans, breu-branco-da-várzea - Protium unifolium, ingá - Inga distinta, louro-da-várzea - Nectandra amazonium, sumaúma - Ceiba pentandra e buriti - Mauritia flexuosa (Santos et al., 2003).

\section{Metodologia de coleta e análise de dados}

A coleta de dados foi realizada em uma área de 200 hectares de floresta tropical primária de terra-firme que sofreu exploração florestal seletiva em 1997, pela empresa Perachi Ltda, sob a orientação da Embrapa Amazônia Oriental.

A área foi dividida em 2 blocos iguais de 100ha, onde foram inventariadas árvores de espécies comerciais com DAP > $25 \mathrm{~cm}$ (bloco 2) e DAP > 45cm (bloco 1).

Dessa exploração originaram-se diversas clareiras, dentre as quais nove foram utilizadas para a realização deste estudo (Figura 2), com áreas variando entre $231 \mathrm{~m} 2$ e $748 \mathrm{~m} 2$ sendo que as áreas das clareiras foram calculadas considerando que as mesmas fossem retangulares. Com auxílio de equipamentos topográficos como trena e bússola, cada clareira teve seu centro determinado. 
Figura 2 - Desenho esquemático da disposição das nove clareiras selecionadas para estudo no Campo Experimental da Embrapa Amazônia Oriental em Moju - PA.

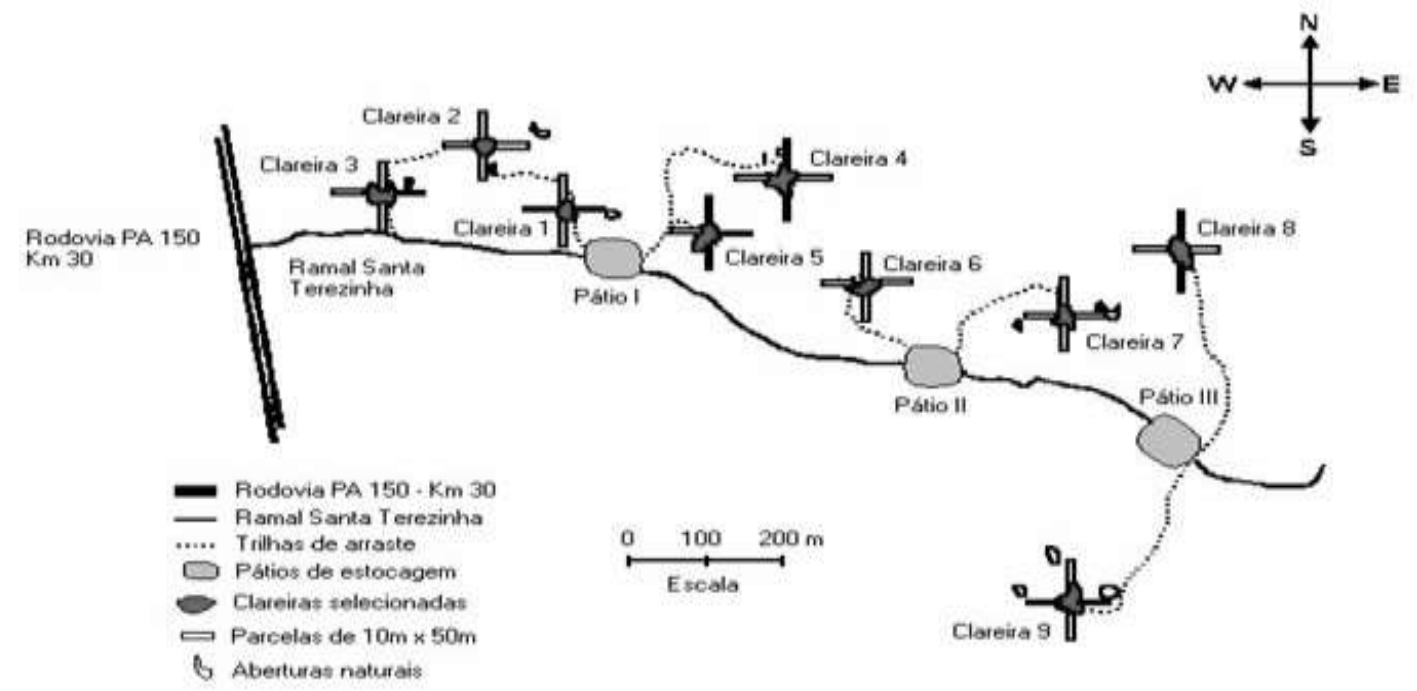

Fonte: Autores.

O presente estudo teve início em março de 1998, compondo a linha de pesquisa do projeto "Rendimento Sustentável em Floresta Tropical Manejada”, de responsabilidade do Grupo de pesquisas MANEJO FCAP, que recebeu financiamento do CNPq (Conselho Nacional de Desenvolvimento Científico e Tecnológico) e SECTAM (Secretaria Executiva de Ciência, Tecnologia e Meio Ambiente)/FUNTEC (Fundo Estadual de Ciência e Tecnologia) e o apoio logístico da Embrapa Amazônia Oriental/UFRA e moradores da região.

As atividades de coleta de dados foram realizadas trimestralmente, especificamente a partir do 20o dia dos meses de março, junho, setembro e dezembro. Para este estudo, os dados analisados são correspondentes ao período de junho de 1998 a junho de 2001 (três anos).

Em cada clareira foram marcadas quatro faixas de 10m x 50m (Figura 3), nas direções Norte (N), Sul (S), Leste (E) e Oeste (W), divididas em parcelas de $10 \mathrm{~m}$ x 10m, onde foi realizado um inventário florestal de árvores com DAP > 5cm, correspondendo ao nível II de abordagem. O nível I de abordagem consistiu na medição de plantas com altura total > $10 \mathrm{~cm}$ e DAP $<5 \mathrm{~cm}$, em parcelas de $2 \mathrm{~m}$ x 2m localizadas nas sub-parcelas de 10m x 10m do nível II de abordagem. 
Figura 3 - Desenho esquemático da distribuição das parcelas amostrais para o nível I de abordagem.

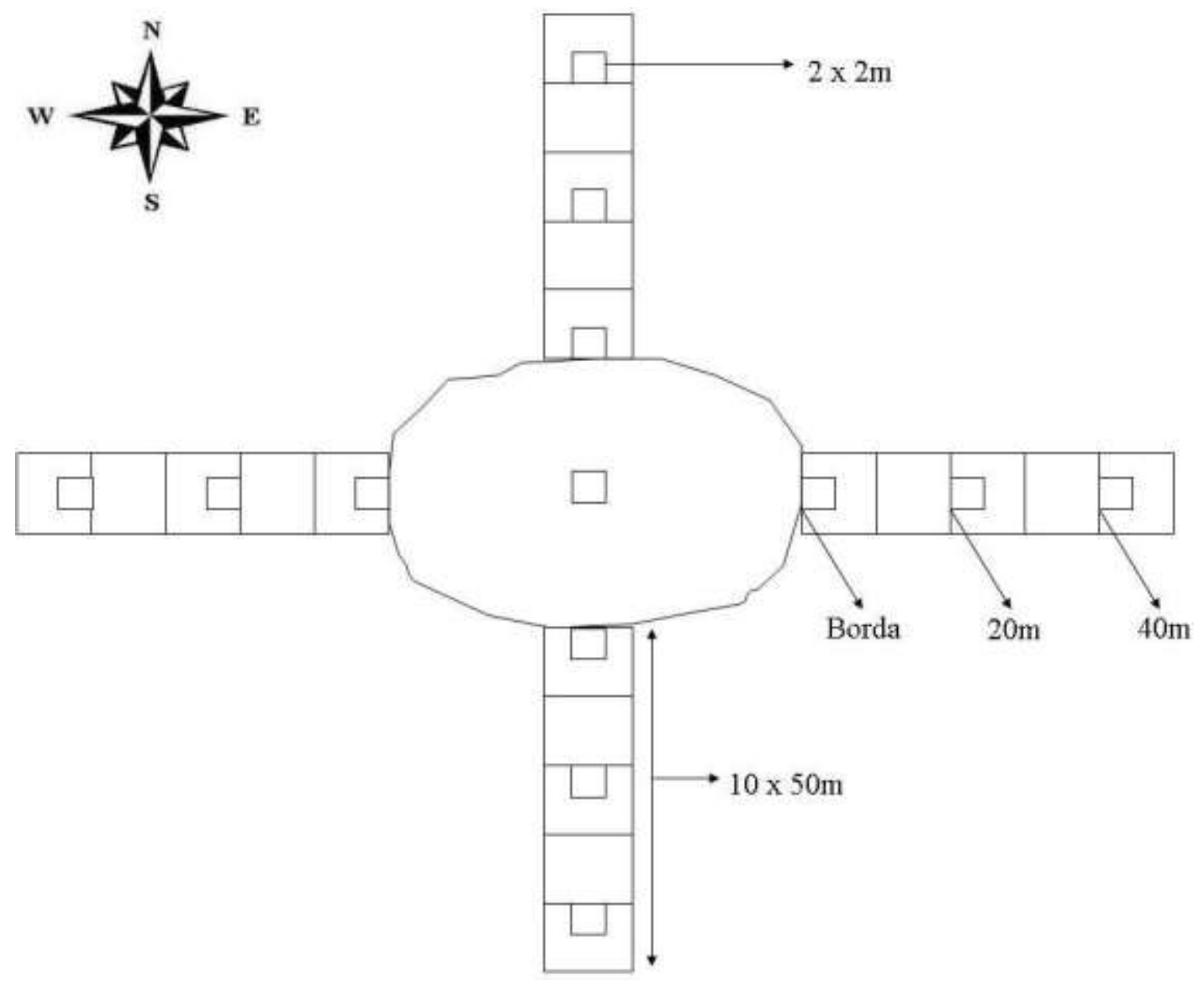

Fonte: Autores.

O comportamento da regeneração natural (TR) de Protium pallidum Cuatrec. foi avaliado pela equação proposta por Mory (2000), a qual é definida como a razão entre a abundância absoluta resultante do processo dinâmico de regeneração natural (recrutamento, crescimento e mortalidade) e a abundância absoluta no início do estudo, em percentagem, e representada pela expressão:

Onde:

$\operatorname{Tr}$ - taxa de regeneração natural em percentagem;

A - abundancia absoluta

Ao - abundância absoluta no início do período;

A1 - abundância absoluta no final do período;

$\mathrm{A} 1=\mathrm{Ao}+\mathrm{ni}-\mathrm{ns}$

Onde:

ni - número de indivíduos que ingressaram no estudo, por germinação ou mudança de categoria de tamanho devido ao crescimento;

ns - número de indivíduos que saíram do estudo, por morte ou mudança de categoria de tamanho devido ao crescimento.

O comportamento do ingresso, mortalidade e sobrevivência de Protium pallidum Cuatrec. foi avaliado pelas equações:

$$
\mathrm{I}=\mathrm{ni} / \mathrm{A} 0
$$


$\mathrm{M}=\mathrm{nm} / \mathrm{A} 0$

Onde:

I - taxa de ingresso em percentagem;

$\mathrm{M}$ - taxa de mortalidade em percentagem;

$\mathrm{nm}$ - número de indivíduos que morreram durante o período.

A dinâmica da regeneração natural foi avaliada em relação às direções Norte, Sul, Leste e Oeste das clareiras, bem como em relação às distâncias do centro das clareiras para o interior da floresta, ou seja, nas bordas das clareiras e a 20m e $40 \mathrm{~m}$ das bordas para o sub-bosque. Também foram registrados valores para o período de três anos de monitoramento e a cada ano isoladamente.

O DAP, sempre que possível, foi medido a $1,30 \mathrm{~m}$ a partir da superfície do solo. Na ocorrência de sapopemas, deformações ou irregularidades nos troncos, o ponto de medição passou a ser logo acima da anormalidade. Para se evitar erros nas medições posteriores, pintou-se uma faixa com tinta à base de óleo vermelha no ponto da medição.

Todos os indivíduos de Protium pallidum Cuatrec. foram identificados com pequenas placas de alumínio em que constam nível de abordagem (I ou II) indicando o estudo da regeneração natural ou povoamento adulto, clareira, direção, parcela e número da planta, de acordo com o exemplo a seguir:

Exemplo:

Para um melhor entendimento da análise realizada em relação ao período, deve-se considerar que em três anos de estudo foram realizadas treze medições trimestrais. Observando o esquema a seguir (Figura 4), nota-se que o Ano 1 se refere à comparação da $5^{\text {a }}$ medição com a $1^{\mathrm{a}}$ medição, o Ano 2 refere-se à comparação da $9^{\mathrm{a}}$ medição com a $5^{\mathrm{a}}$ e o Ano 3 compara a $13^{\mathrm{a}}$ medição com a $9^{\mathrm{a}}$. Por fim, a análise de três anos compara a $13^{\mathrm{a}}$ medição com a $1^{\mathrm{a}}$ medição, ou seja, a TR encontrada no final do estudo (Figura 4).

Figura 4 - Desenho esquemático das medições durante os três anos.

3 Anos

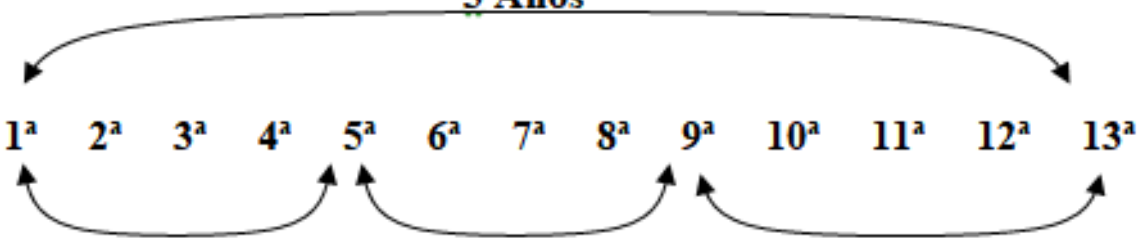

Ano 1

Ano 2

Ano 3

Fonte: Autores.

Os resultados foram analisados no programa SYSTAT ${ }^{\circledR} 10$ for Windows (SAS Institute Inc. in the USA and other countries. ® indicates USA registration), com Análise de Variância de três fatores (4 direções, 3 distâncias e os 4 anos) sobre as variáveis dependentes: Taxa de Regeneração Natural (TR\%), Ingresso (I\%) e Mortalidade (M\%), para o Nível I de abordagem; e sobre o Ingresso (I\%), Mortalidade (M\%) e crescimento diamétrico da espécie, através do Incremento Corrente Anual em diâmetro, para o Nível II de abordagem. Também foi utilizado o tamanho das clareiras como covariável.

\section{Resultados e Discussão}

Os resultados da análise de variância de três fatores referentes aos dados de regeneração natural estão expressos na 
Tabela 1.

Tabela 1 - Análise de Variância dos dados referentes às Taxas de Regeneração Natural $(\%)$ de Protium pallidum $\left(\mathrm{r}^{2}=0,135\right)$.

\begin{tabular}{lcccc}
\hline Fonte de variação & Grau de liberdade & F & P & Significância \\
\hline Distância & 2 & 0,861 & 0,424 & NS* \\
Cardeal & 3 & 0,959 & 0,412 & NS \\
Ano & 2 & 0,643 & 0,526 & NS \\
Dist*Card & 6 & 1,412 & 0,210 & NS \\
Dist*Ano & 4 & 2,768 & 0,028 & S** \\
Card*Ano & 6 & 0,474 & 0,828 & NS \\
Dist*Card*Ano & 12 & 1,369 & 0,180 & NS \\
Tam. da Clar. & 1 & 0,020 & 0,887 & NS \\
Erro & 287 & & & \\
\hline
\end{tabular}

* - Não significante. ** - Significante. Fonte: Autores.

Como as clareiras haviam sido classificadas em diferentes classes de tamanho, utilizou-se nas análises o tamanho das clareiras como co-variável. No entanto, essa covariância não apresentou influência significativa sobre os resultados, sendo os mesmos não considerados no que diz respeito aos resultados e discussões.

TR (\%) de Protium pallidum Cuatrec. nas direções norte, sul, leste e oeste.

Não houve diferenças significativas nos valores de TR encontradas em função das direções (F[0,959], P[0,412], Tabela 1), embora se tenha encontrado um valor médio maior para a direção Sul (Figura 5).

Figura 5 - Médias de Taxa de Regeneração Natural de Protium pallidum em função dos pontos cardeais.

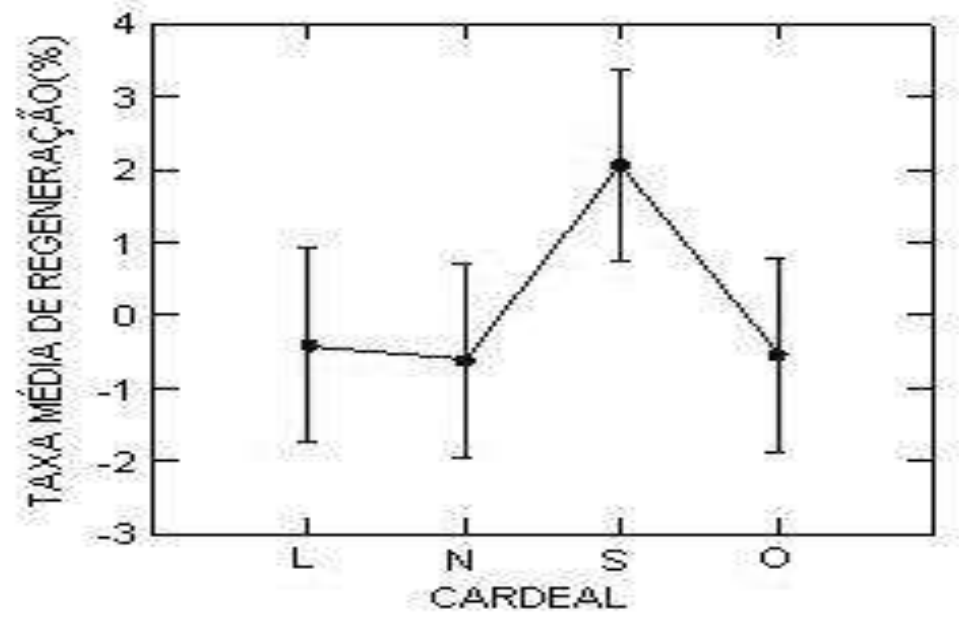

Fonte: Autores. 
TR (\%) de Protium pallidum Cuatrec. nas distâncias do centro das clareiras.

Nos valores de TR encontradas em função das distâncias também não houve diferenças significativas ( $F[0,861]$, $\mathrm{P}[0,424]$, Tabela 1), no entanto, observa-se pequeno aumento à medida que se adentra no sub-bosque (Figura 6).

Figura 6 - Médias de Taxa de Regeneração Natural de Protium pallidum em função das distâncias do centro das clareiras para o interior do sub-bosque.

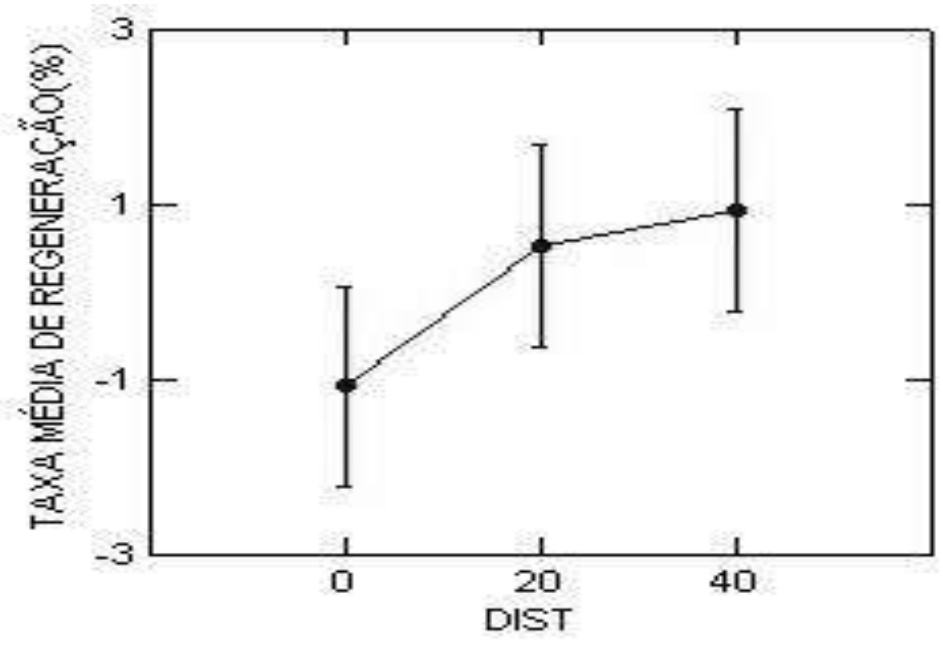

Fonte: Autores.

\section{Comportamento da TR(\%) De Protium pallidum Cuatrec. Nos Três Anos De Monitoramento.}

Não houve diferenças significativas nos valores de TR encontradas em função dos três anos de estudo (F[0,646], $\mathrm{P}[0,526]$, Tabela 1) (Figura 7), embora se tenha registrado um valor negativo ao final do primeiro ano e uma variação marginalmente significativa (muito próxima da significância) entre o $1^{\circ}$ e $2^{\circ}$ anos, sugerindo que o índice de ingressos foi maior que o de egressos. Essa variação diminuiu entre o $2^{\circ} \mathrm{e} 3^{\circ}$ anos de estudo, demonstrando ligeira tendência à estabilização.

Figura 7 - Médias de Taxa de Regeneração Natural de Protium pallidum em função do período de três anos de estudos.

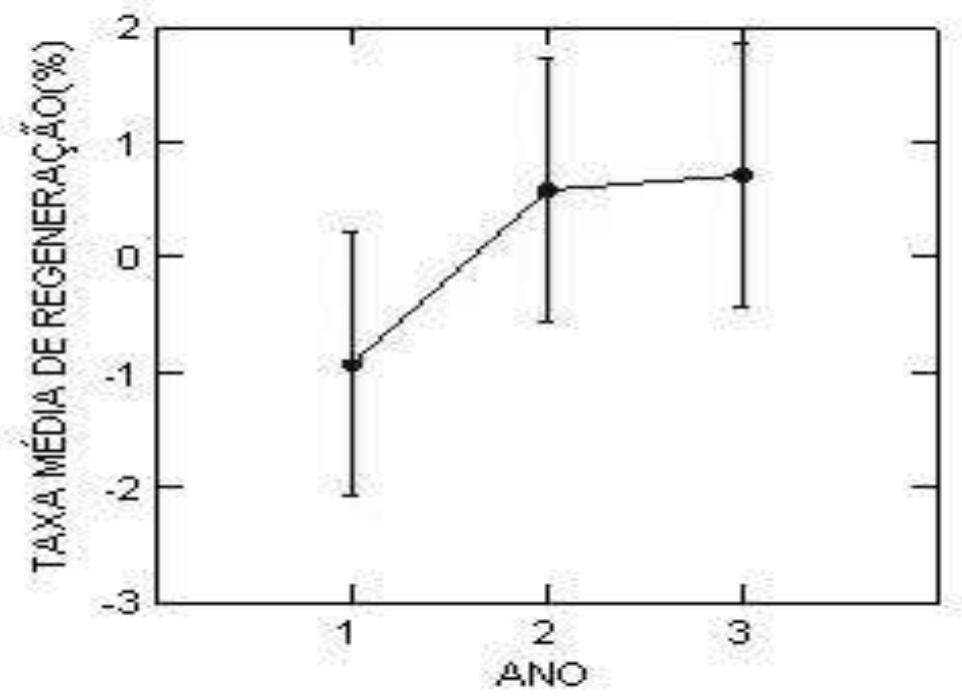

Fonte: Autores.

A interação entre os fatores Distância x Anos mostrou diferença significativa entre os valores de TR (F[2,768], 
$\mathrm{P}[0,028]$, Tabela 1), isso talvez possa ser explicado pelo fato de, no primeiro ano (logo após a exploração) a maior presença de radiação solar fez com que a regeneração fosse mais eficiente. Após isso, supõe-se que no decorrer do $2^{\circ}$ e $3^{\circ}$ anos, tenha ocorrido um adensamento no sub-bosque, provocando um pequeno fechamento no dossel florestal nas parcelas mais distantes do centro (20m e 40m) e tendência à estabilização, tanto em função das distâncias, quanto em função do tempo.

Malheiros (2001) constatou em seu trabalho que o eixo Leste-Oeste oferece maior quantidade e qualidade de radiação fotossinteticamente ativa para as plantas. Isso contrasta com os resultados encontrados para TR, onde foram registrados valores médios maiores na direção Sul, onde a radiação é menor. Esse resultado reforça o conceito de espécie tolerante à sombra, para esta espécie, segundo o qual as plantas não dependem de radiação direta para seu desenvolvimento, no entanto se beneficiam com ela.

Em termos gerais, Protium pallidum se regenerou melhor no eixo norte-sul. A parcela localizada no centro das clareiras não apresentou nenhum indivíduo.

Brokaw (1985), observando a ocorrência de espécies regenerando em ambientes aparentemente desfavoráveis em termos de intensidade luminosa, registrou a ocorrência de 53 espécies consideradas tolerantes, regenerando em clareiras de diferentes tamanhos.

Esse comportamento pode ser típico, se não de todas as espécies tolerantes, mas de algumas, mostrando a capacidade de adaptar-se a ambientes diversos. Ressalta-se o fato das espécies tolerantes suportarem ambientes com baixa quantidade de radiação, em vez de considerar que tenham melhor desempenho em ambientes sombreados. Portanto, fazer ensaios para saber em que nível máximo de radiação essas espécies sobrevivem seria responder qual é o nível de abertura suportado por essas espécies, principalmente em atividades que lidam com abertura do dossel, como, por exemplo, a exploração madeireira, tratamentos como desbastes, etc (Serrão et al, 2003).

Vasconcelos (2004) encontrou resultados semelhantes ao estudar a espécie Rinorea guianensis Aublet. Essa espécie também apresentou resultados bastante aproximados para as quatro direções nas mesmas condições de estudo, demonstrando uma TR que não expressou relação com o mesmo gradiente de radiação.

Nemer (2003), ao estudar o comportamento da população de Eschweilera odora (Poepp.) Miers, nessa mesma área e nas mesmas condições, verificou não haver qualquer relação ou tendência entre os valores de TR e as direções Norte, Sul, Leste e Oeste, nem tampouco com as distâncias a partir do centro das clareiras. Dessa forma, depreende que durante o período estudado, E. odora não dependeu dos microambientes provocados pela formação de clareiras para germinar suas sementes.

Esses estudos confirmam que a regeneração das espécies Eschweilera odora (Poepp.) Miers, Rinorea guianensis Aublet e Protium pallidum Cuatrec. se comportam de maneira semelhante ao serem analisadas nas mesmas condições de luminosidade e no mesmo período de tempo, em relação às direções e às distâncias do centro das clareiras.

Nappo et al. (2005) encontrou um aumento de 48,46\% na abundância da regeneração no sub-bosque de um povoamento de Mimosa scabrella Bentham, em seu estudo de três anos sobre a dinâmica em uma área minerada no Estado de Minas Gerais. Esse comportamento de indivíduos é responsável pelas elevadas taxas de ingresso nas menores classes diamétricas do estrato arbóreo da floresta, sendo estes indivíduos denominados "edificadores", ou seja, responsáveis pela manutenção da estrutura e fisionomia da floresta (Jardim, 1990).

Em estudos realizados em uma floresta de galeria em Itutinga (MG), Corrêa \& Berg (2002) observaram para a área total amostrada, aumento do número de indivíduos de 0 a $5 \mathrm{~cm}$ de diâmetro de Xylopia brasiliensis. Esse fato pode estar relacionado a duas possibilidades: a população de X. brasiliensis está aumentando, mas isto ainda não se refletiu nos indivíduos adultos e sub-adultos, ou se trata apenas de uma flutuação cíclica do número de indivíduos menores.

Resultados semelhantes foram encontrados por Felfili (1995), estudando a dinâmica de comunidade de uma floresta de galeria no Brasil central. O grande número de plantas mortas na fase inicial de desenvolvimento dos indivíduos é uma das 
principais características das espécies tolerantes, pois para apresentar a distribuição diamétrica decrescente ou em forma de "J invertido", é necessário que a mortalidade dos indivíduos seja constante à medida em que se aumente a classe diamétrica, compensada por um constante ingresso.

Segundo Mory e Jardim (2001), espécies tolerantes à sombra se caracterizam pelo contínuo recrutamento, devido à grande disponibilidade de sementes com rápida germinação, que não permite acúmulo no banco de sementes. Entretanto, apresentam alta mortalidade, principalmente dos menores indivíduos, que têm menos chances competitivas em relação aos maiores, o que justifica sua distribuição exponencial decrescente.

\section{Ingresso e mortalidade}

Os resultados da análise de variância de três fatores para os dados referentes às Taxas de Ingresso (\%) e Mortalidade (\%) estão expressos na Tabelas 2 e 3.

Tabela 2 - Análise de Variância dos dados referentes às Taxas de Ingresso (\%) de Protium pallidum para o Nível I de abordagem (para $\left.\mathrm{r}^{2}=0,101\right)$.

\begin{tabular}{lcccc}
\hline Fonte de variação & GL & F & P & Significância \\
\hline Distância & 2 & 0,045 & 0,956 & NS* \\
Cardeal & 3 & 0,970 & 0,407 & NS \\
Ano & 2 & 0,045 & 0,956 & NS \\
Dist*Card & 6 & 0,891 & 0,502 & NS \\
Dist*Ano & 4 & 1,294 & 0,272 & NS \\
Card*Ano & 6 & 0,891 & 0,502 & NS \\
Dist*Card*Ano & 12 & 1,109 & 0,352 & NS \\
Tam. da Clar. & 1 & 0,082 & 0,775 & NS \\
Erro & 287 & & & \\
\hline
\end{tabular}

* - Não significante; ** - Significante. Fonte: Autores. 
Tabela 3 - Análise de Variância dos dados referentes à Taxa de Mortalidade (\%) de Protium pallidum para o Nível I de abordagem (para $\left.\mathrm{r}^{2}=0,162\right)$.

\begin{tabular}{lcccc}
\hline Fonte de variação & GL & F & P & Significância \\
\hline Distância & 2 & 2,499 & 0,084 & NS* \\
Cardeal & 3 & 0,549 & 0,649 & NS \\
Ano & 2 & 2,227 & 0,110 & NS \\
Dist*Card & 6 & 1,647 & 0,134 & NS \\
Dist*Ano & 4 & 3,875 & 0,004 & S** \\
Card*Ano & 6 & 1,375 & 0,224 & NS \\
Dist*Card*Ano & 12 & 0,826 & 0,623 & NS \\
Tam. da Clar. & 1 & 0,744 & 0,389 & NS \\
Erro & 287 & & & \\
\hline
\end{tabular}

* - Não significante; ** - Significante. Fonte: Autores.

\section{Ingresso (\%) de Protium pallidum Cuatrec. em relação as direções Norte, Sul, Leste e Oeste.}

Embora tenham se destacado na direção Sul, não houve diferenças significativas nos valores de taxa de ingresso em função das direções do centro das clareiras (F[0,970], P[0,407], Tabela 2) (Figura 8).

Figura 8 - Médias de Taxa de Ingresso de Protium pallidum em função das direções do centro das clareiras.

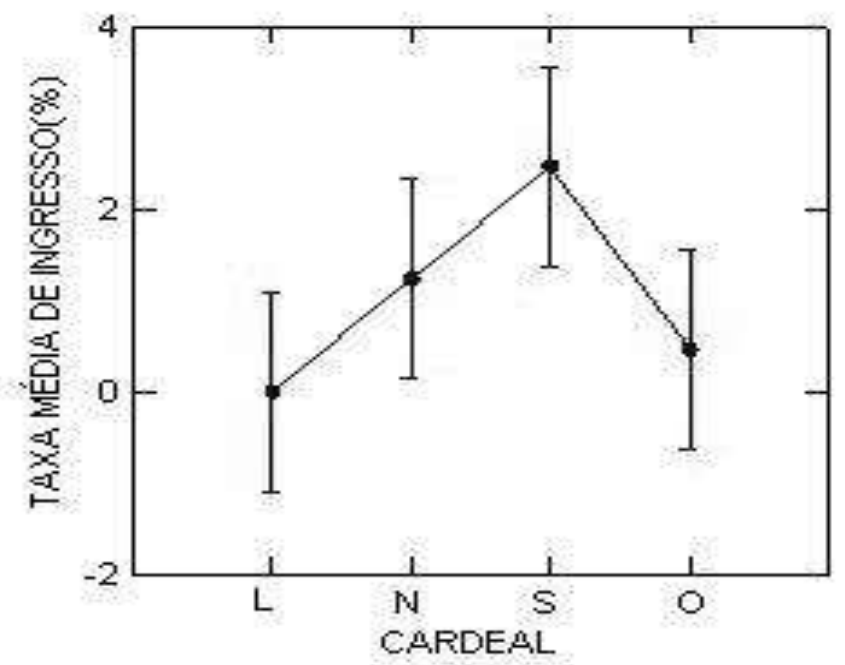

Fonte: Autores.

Ingresso (\%) de Protium pallidum Cuatrec. em relação as distâncias do centro das clareiras.

Não houve diferenças significativas nos valores de taxa de ingresso em função das distâncias do centro das clareiras (F[0,045], P[0,956], Tabela 2) (Figura 9). 
Figura 9 - Médias de Taxa de Ingresso de Protium pallidum em função das distâncias do centro das clareiras.

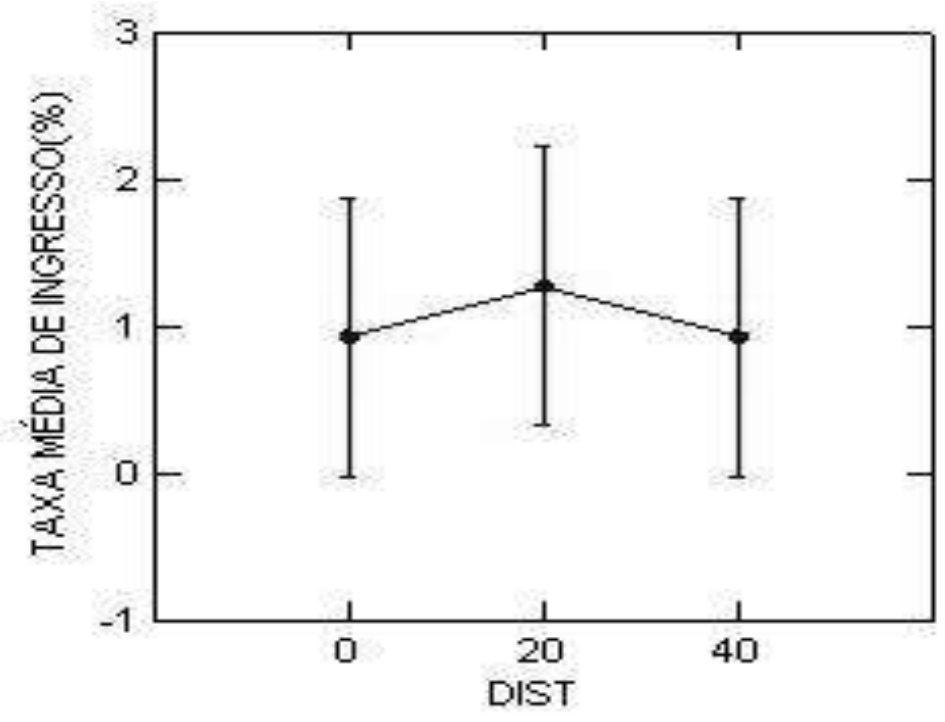

Fonte: Autores.

Comportamento do Ingresso (\%) de Protium pallidum Cuatrec. nos três anos de monitoramento.

Os valores de taxa de ingresso também não apresentaram diferenças significativas em relação aos três anos de estudos (F[0,045], P[0,956], Tabela 2) (Figura 10).

Figura 10 - Médias de Taxa de Ingresso de Protium pallidum em função do período de três anos de estudos.

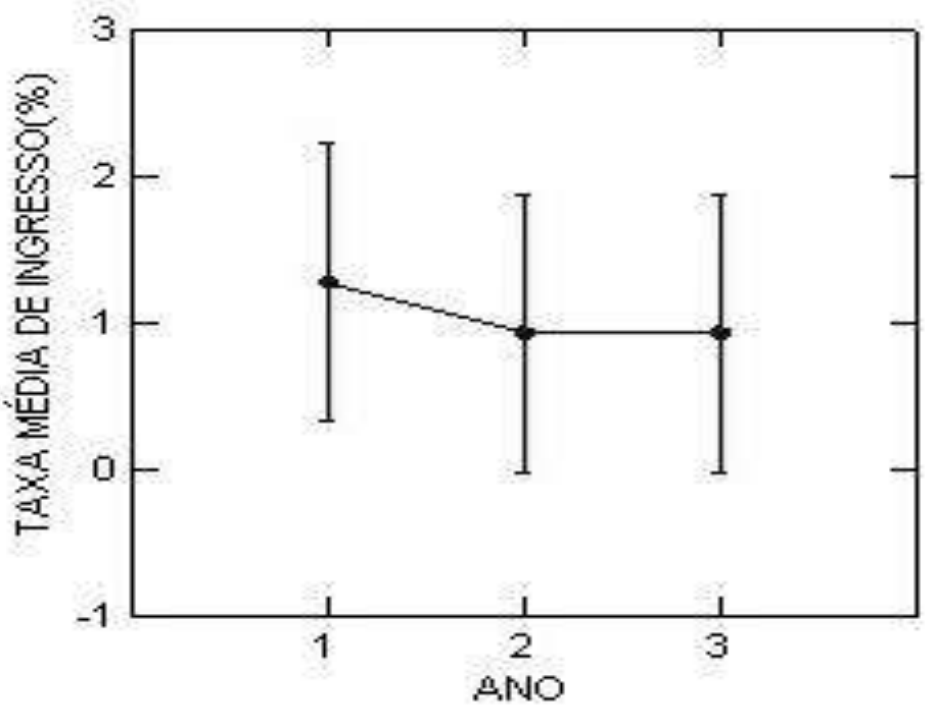

Fonte: Autores.

\section{Mortalidade (\%) de Protium pallidum Cuatrec. em relação as direções Norte, Sul, Leste e Oeste.}

Embora se observe pequeno destaque na direção Norte, não houve diferenças significativas nos valores de taxa de mortalidade em função das direções do centro das clareiras (F[0,549], P[0,649], Tabela 3) (Figura 11). 
Figura 11 - Médias de Taxa de Mortalidade de Protium pallidum em função das direções do centro das clareiras.

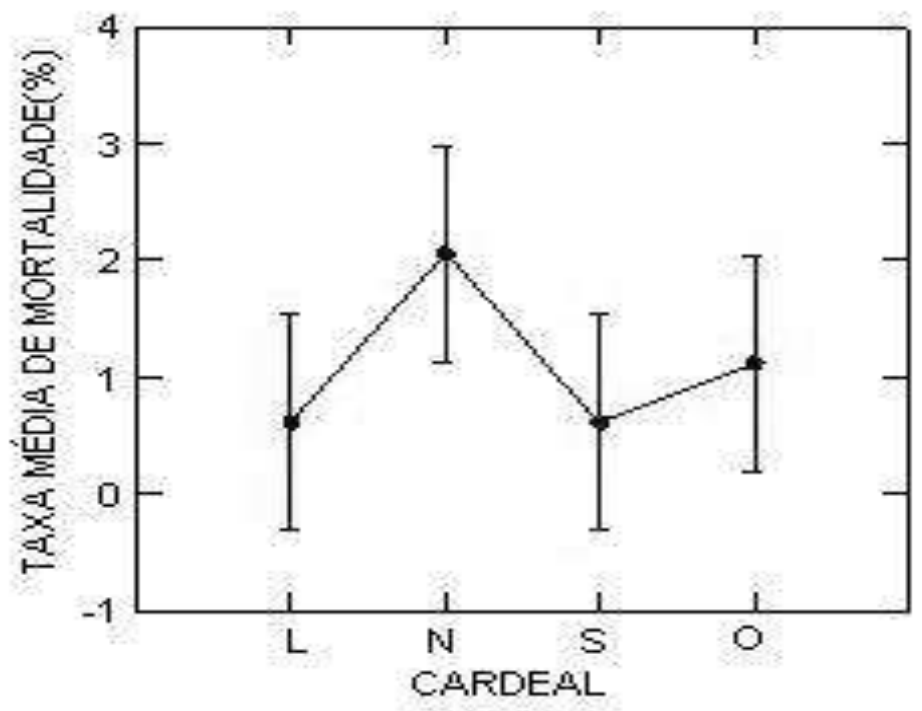

Fonte: Autores.

\section{Mortalidade (\%) de Protium pallidum Cuatrec. em relação as distâncias do centro das clareiras.}

Não houve diferenças significativas nos valores de taxa de mortalidade em função das distâncias do centro das clareiras $(\mathrm{F}[2,499], \mathrm{P}[0,084]$, Tabela 3), contudo podemos observar diminuição da mortalidade à medida que se adentra no sub-bosque (Figura 12).

Figura 12 - Médias de Taxa de Mortalidade de Protium pallidum em função das distâncias do centro das clareiras.

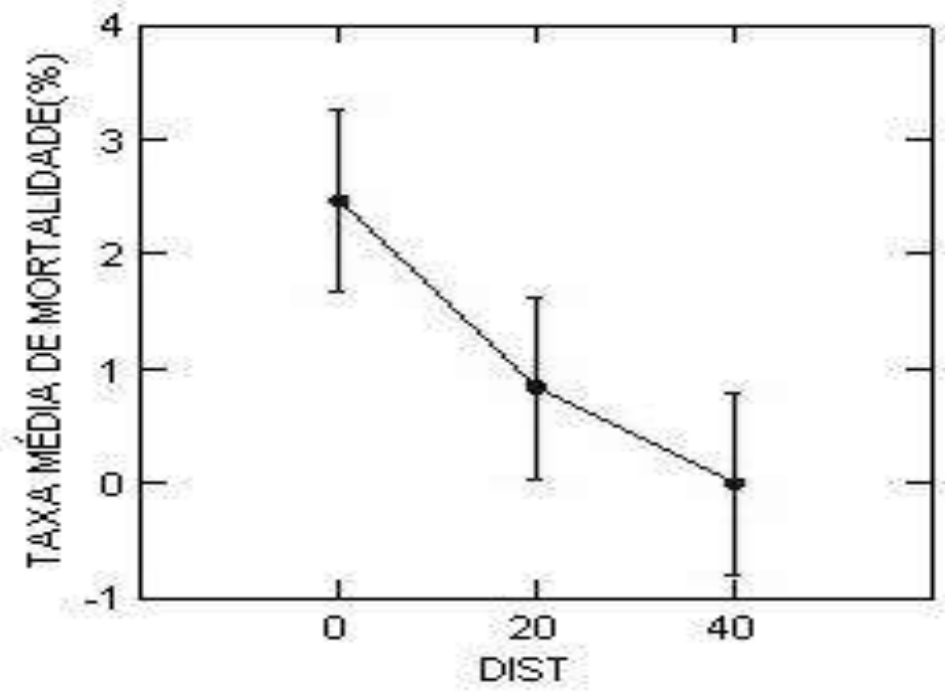

Fonte: Autores.

\section{Comportamento da Mortalidade (\%) de Protium pallidum Cuatrec. nos três anos de monitoramento.}

Os valores de taxa de mortalidade também não apresentaram diferenças significativas em relação aos três anos de estudos (F[2,227], P[0,110], Tabela 3), entretanto, nota-se diminuição da mortalidade entre o $1^{\circ}$ e $2^{\circ}$ ano, mostrando tendência à estabilização ao final do $3^{\circ}$ (Figura 13). 
Figura 13 - Médias de Taxa de Mortalidade de Protium pallidum em função do período de três anos de estudos.

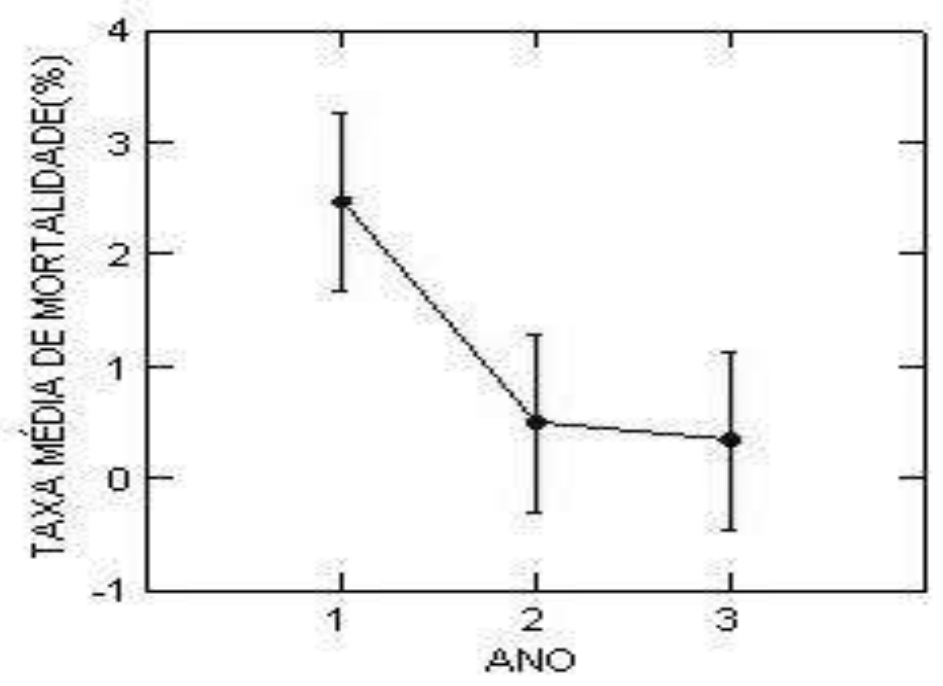

Fonte: Autores.

Os resultados mostram que apesar de terem apresentado variações de níveis diferentes no decorrer do estudo, os valores de taxa de ingresso foram positivos em relação aos valores de taxa de mortalidade, isto se reflete na taxa de regeneração vista anteriormente.

As diferenças significativas vistas nos valores de mortalidade em relação à interação dos fatores Distância x Anos ( $\mathrm{F}[3,875], \mathrm{P}[0,004]$, Tabela 3 ) podem ser explicadas pelo fato de que na área mais próxima e no período logo após o distúrbio, a mortalidade tende a ser maior, diminuindo ao longo do tempo e se estabilizando no processo de sucessão.

Carvalho et al. (1999), em trabalho realizado em uma floresta próxima a Manaus - AM verificaram que o ingresso (ou recrutamento) cresceu com o tempo na floresta explorada, enquanto a mortalidade, ainda que mais baixa do que o recrutamento, foi muito alta imediatamente após a exploração, baixando devagar ate o quinto ano após a exploração, mantendo-se com taxas baixas até o final do período estudado.

No estudo da dinâmica de regeneração natural após a extração seletiva na Floresta de Tapajós, Silva (1989) obteve um decréscimo de $34 \%$ no total de plântulas, em um período de 6 anos. Considerou ainda que essa flutuação na população de plântulas pode ser resultado de diferentes frequências de frutificação, manifestadas pelas diferentes espécies.

Silva et al. (1999), também na Floresta Nacional do Tapajós, concluíram que a mortalidade aumentou entre o primeiro e segundo período de observação (1,8\% ao ano), porém nos 11 anos de observações (13 após a colheita) a taxa de mortalidade foi de $2,2 \%$ ao ano.

Em outros trabalhos (Mesquita, 1998; Marquis et al, 1986) foi comprovado que a remoção do dossel é benéfica para a maioria das espécies florestais, obtendo a aceleração do crescimento e aumento na taxa de sobrevivência das mesmas.

Dependendo do grau de abertura, pode ocorrer maior ingresso do que crescimento, ou vice-versa. Portanto, trabalhos que venham contribuir para o melhor manejo de espécies e, consequentemente, de florestas, devem ser colocados em prática para gerar informações que serão subsídios ao manejo de florestas na Amazônia (Serrão, 2003).

Em estudos sobre dinâmica florestal na região amazônica, Mendonça (2003) registrou que a maioria das espécies, quando consideradas separadamente, tiveram a taxa de recrutamento mais alta do que a de mortalidade na área explorada no período estudado, enquanto que na área não explorada um grande número de espécies mostrou a mesma taxa, tanto em recrutamento como em mortalidade, embora algumas outras apresentassem grandes diferenças. Este mesmo autor cita ainda que, em geral, na floresta não explorada as diferenças entre recrutamento e mortalidade não foram importantes, mas levemente 
balanceadas durante o período estudado.

Na área estudada por Pereira et al. (2000), entre os anos 1986, 1989, 1991 e 1993, as taxas de recrutamento foram superiores às taxas de mortalidade. Entre 1986 e 1989, e entre 1991 e 1993, a mortalidade foi maior do que o recrutamento.

Vasconcelos (2004), ao estudar a dinâmica populacional de Rinorea guianensis Aublet, encontrou valores de ingresso e mortalidade de $307,96 \%$ e $25,87 \%$, respectivamente. Nascimento (2003) encontrou valor menor para mortalidade (37,28\%), ao realizar o mesmo estudo com Lecythis idatimon Aublet, nas mesmas condições.

Observa-se que os valores expressos nos resultados são valores médios decorrentes das análises estatísticas. É por esse motivo que quando os mesmos foram comparados com resultados de outros autores, não se utilizaram valores percentuais.

Vale ressaltar que os resultados acima explicam cerca de 10 a $16 \%$ das variações ocorridas para o nível de regeneração natural, sendo conveniente a avaliação de novos parâmetros e/ou até a aplicação de outros métodos para a avaliação dessa dinâmica.

\section{Conclusão}

A regeneração natural de Protium pallidum Cuatrec. não sofreu influência das quatro direções, nem tampouco das distâncias do centro das clareiras.

A grande variação de ingresso e mortalidade da regeneração natural indicou que Protium pallidum Cuatrec. tem um comportamento de espécie tolerante à sombra.

O conhecimento sobre a dinâmica das espécies florestais sejam elas tolerantes ou não, pode ser muito bem utilizado nos Planos de Manejo Florestal Sustentável, pois além de colaborar com um melhor planejamento dos ciclos de corte, auxiliam também no planejamento dos tratamentos silviculturais, tanto antes como após a exploração, pois podem interferir na escolha das espécies para possíveis desbastes.

A interação significativa entre os fatores distância $x$ anos não foi explicada com eficiência neste trabalho, sendo que um maior período de monitoramento na área de estudo é de fundamental importância para que a dinâmica não só dessa espécie, bem como de outras sejam melhor avaliada.

\section{Referências}

Brokaw, N. V. L. (1985). Gap-phase regeneration in a tropical forest. Ecology, 66: 682-687. https://doi.org/10.2307/1940529.

Brokaw, N. V. L., \& Scheiner, S. M. (1989). Species composition in gaps and structure of a tropical forest. Ecology, 70, 538-541. https://esajournals.onlinelibrary.wiley.com/doi/abs/10.2307/1940196

Brown, N. (1993).The implications of climate and gap microclimate for seedling growth conditions in a Bornean lowland rain forest. Journal of Tropical Ecology, 9, 153-168. 10.1017/S0266467400007136

Canham, C. D. (1989). Different responses to gaps among shadetolerant tree species. Ecology, 70, 548-550. https://doi.org/10.2307/1940200

Carneiro, F. S., Ruschel, A. R., Freitas, L. J. M., Pinheiro, K. A. O., D'arace, L. M. B., \& Maestri, M. P. (2019). Resiliência do volume de madeira de espécies comerciais em diferentes áreas experimentais na Amazônia Oriental. Revista Ibero Americana de Ciências Ambientais, 10(6), 15-31. http://doi.org/10.6008/CBPC2179-6858.2019.006.0003

Carvalho, J. O. P. (1999). Dinâmica de florestas naturais e sua implicação para o manejo florestal. In: Simpósio Silvicultura Na Amazônia Oriental: Contribuições do Projeto EMBRAPA, Anais. Belém: EMBRAPA, 174-179. https://www.embrapa.br/busca-de-publicacoes/-/publicacao/394985/dinamica-deflorestas-naturais-e-sua-implicacao-para-o-manejo-florestal

Clark, D. B. (1990). The role of disturbance in the degeneration of neotropical moist forests. In: Bawa, K. B., Hadley, M. (Eds.). Reproductive ecology of tropical forest plants. Paris: UNESCO. p. 291-315.

Corrêa, B. S. \& Berg, E. V. D. (2002). Estudo da dinâmica da população de xylopia brasiliensis Sprengel em relação a parâmetros populacionais e da comunidade em uma floresta de galeria em Itutinga, MG, Brasil. Revista Cerne, 8(10), 01-012. https://www.redalyc.org/articulo.oa?id=74408101

Denslow, J. S. (1980). Gap partitioning among tropical rainforest trees. Biotropica, 12, 47-55. https://doi.org/10.2307/2388156 
Felfili, J. M. (1995). Growth, recruitment and mortality in the Gama gallery forest in central Brazil over a six-year period. Journal of Tropical Ecology, Cambridge, 11(1), 67-83. https://doi.org/10.1017/S0266467400008415

Jardim, F. C. S. Mortalidade e crescimento na floresta equatorial de terra-firme. Boletim do Museu Paraense Emílio Goeldi, Série Botânica, 6(2), 222-234, 1990.

Johns, A. G. (1997). Timber production and biodiversity conservation in tropical rain forests. Cambridge University Press. p. 210. https://doi.org/10.1017/CBO9780511525827.

Malheiros, M. A. B. (2001). Caracterização do fluxo de radiação fotossinteticamente ativa, irradiância espectral e relação vermelho:vermelho extremo em clareiras da exploração florestal seletiva, em Moju-Pará, Brasil, 93f. Dissertação (Mestrado) - FCAP. Belém.

Marquis, R. J., Yung, H. J. \& Braker, H. E. (1986). The influence of undertory vegetation cover on germination and seedlings establishment in a tropical lawland wet forest. Biotropica. 18(4): 273-278. https://doi.org/10.2307/2388569.

Mendonça, A. C. A. (2003). Caracterização e simulação dos processos dinâmicos de uma área de floresta tropical de terra firme utilizando matrizes de transição. Dissertação (Mestrado em Ciências Florestais) - Universidade Federal do Paraná, Curitiba - PR. https://hdl.handle.net/1884/2507

Mesquita, R. C. G. (1998). O impacto da remoção do dossel de uma mata secundária no crescimento de duas espécies de interesse econômico da Amazônia. In: Gascon, C. \& Montinho, P. Floresta Amazônica: Dinâmica, Regeneração e Manejo. Manaus. 261-274.

Mory, A. M., Jardim, F. C. S. (2001). Comportamento de Eschweilera odora (Popp.) Miers (Matamata-branco) em diferentes níveis de desbaste por anelamento. Revista de Ciências Agrárias, Belém, (36), 29-53, https://cepnor.ufra.edu.br/index.php?journal=ajaes\& page $=$ article\&op=view\&path $\% 5 \mathrm{~B} \% 5 \mathrm{D}=1985 \&$ path $\% 5 \mathrm{~B} \% 5 \mathrm{D}=606$

Mory A. M. (2000). Comportamento de espécies arbóreas em diferentes níveis de desbaste por anelamento. 100f. Dissertação (Mestrado em Ciências Florestais) - FCAP, Belém.

Nappo, M. E., Griffith, J. J., Martins, S. V., Marco Júnior, P., Souza, A. L., \& Oliveira Filho, A. T. (2005). Dinâmica da estrutura diamétrica da regeneração natural de espécies arbóreas e arbustivas no sub-bosque de povoamento puro de mimosa scabrella bentham, em área minerada, em poços de caldas, MG. Revista Árvore, Viçosa-MG, 29(1), 35-46. https://doi.org/10.1590/S0100-67622005000100005

Nascimento, Z. P. D. (2003). Dinâmica da população de Lecythis idatimon Aublet em floresta tropical de terra-firme explorada seletivamente. 66f. Dissertação (Mestrado em Ciências Florestais) - Universidade Federal Rural da Amazônia, Belém.

Nemer, T. C. (2003). Dinâmica da população de Eschweilera odora (Popp.) Miers (Matamata-branco) em floresta tropical de terra firme manejada, Moju Pará - Brasil. 76f. Dissertação (Mestrado em Ciências Florestais) - Universidade Federal Rural da Amazônia, Belém.

Pereira, L. C., Higuchi, N. \& Santos, J. (2000). Taxas de mortalidade e recrutamento de espécies arbóreas em florestas manejadas e não manejadas (primárias) de terra firme da região de Manaus. In: Jornada De Iniciação Científica, Vol. 9, Anais. Manaus: INPA, p. 247-250.

Santos, P. L. (1985). Levantamento semidetalhado dos solos e avaliação da aptidão agrícola das terras para cultura de dendê e seringueira. Projeto Moju Pará. Relatório técnico. Rio de Janeiro: Embrapa/SNLCS. 192p. https://www.embrapa.br/busca-de-publicacoes/-/publicacao/674758/levantamentosemidetalhado-dos-solos-e-avaliacao-da-aptidao-agricola-das-terras-do-campo-experimental-do-caldeirao-do-cpaaembrapa-iranduba-amazonas .

Serrão, D. R., Jardim, F. C. S. \& Nemer, T. C. (2003). Sobrevivência de seis espécies florestais em uma área Explorada seletivamente no município de moju, Pará. Revista Cerne, Universidade Federal de Lavras, Lavras. 9(2), 153-163, https://www.researchgate.net/publication/237039520_Sobrev ivencia_de_seis_especies_florestais_em_uma_area_explorada_seletivamente_no_municipio_de_Moju_Para.

Silva, J. N. M., Carvalho, J. O. P., Lopes, J. C. A., Almeida, B. F., Costa, D. H. M., Oliveira, L. C., Vanclay, J. K., \& Skovsgaard, J. P. (1999). Crescimento e produção de uma floresta tropical da Amazônia brasileira treze anos após a exploração. In: Simpósio Silvicultura Na Amazônia Oriental: Contribuições Do Projeto Embrapa, Anais. Belém: EMBRAPA. p.186-189. https://www.embrapa.br/busca-de-publicacoes/-/publicacao/394987/crescimento-e-producao-deuma-florestal-tropical-da-amazonia-brasileira-treze-anos-apos-a-exploracao.

Silva, J. N. M. (1989). The behaviour of the tropical rain forest of the Brazilian Amazon after logging. England. $312 \mathrm{f}$. Thesis. Oxford University.

Vasconcelos, L. M. R. (2004). Avaliação da dinâmica populacional de Rinorea guianensis Aublet (Acariquarana) Violaceae, em uma floresta tropical primária explorada seletivamente, Moju - PA. Dissertação (Mestrado em Ciências Florestais) - Universidade Federal Rural da Amazônia. Belém. 63f.

Vieira, G. \& Hosokawa, R. T. (1989). Composição florística da vegetação da regeneração natural 1 ano após diferentes níveis de exploração de uma floresta tropical úmida. Acta Amazonica, 19, 401-413. https://doi.org/10.1590/1809-43921989191413

Vieira, G. (1995). Dynamics of the remaining plant population in gaps after logging in the Amazon. In: Malik, A. R. A., Nik, A. R., Mohamad, A., See, L. S., Hoy, A. W. H.\& Choon, K. K. (Eds.). Forestry and Forest Products Research: proceeding of the third conference. Malaysia: Forest Research Institute Malaysia. p. 54-67.

Whitmore, T. C. (1991). Tropical rain forest dynamics and its implications for management. In: Gomez-Pompa A., Whitmore T.C. \& Hadley M. Rain forest regeneration and management. Paris: UNESCO. 667-689. 